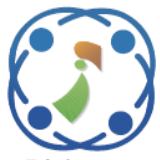

\title{
Design a Linear and Circular Polarization MIMO Antennas Based on Compact Size Configurations with High Isolation and Stable Gain Characteristics for C- Band and WLAN / WiMAX Applications
}

\author{
Qasim Hadi Kareem ${ }^{1 *}$ \\ Malik Jasim Farhan ${ }^{1}$ \\ ${ }^{I}$ Electrical Engineering Department, Mustansiriyah University, Baghdad, Iraq \\ * Corresponding author's Email: qasim.hadi2017@gmail.com
}

\begin{abstract}
This paper presents a compact linear and circular polarization multi-input multi-output (MIMO) antenna for wireless local area network (WLAN) / worldwide interoperability for microwave access (WiMAX) and C-band wireless communication systems. This work has been performed with two configurations of six-port MIMO antennas with a total size of $60 \times 60 \mathrm{~mm}^{2}$ on low-cost FR-4 substrate. There are two rectangular slots - one is situated on the upper part of the circular patch to radiate left hand circular polarization (LHCP) waves, and the other is located on the lower portion of the circular patch to emit right hand circular polarization (RHCP) waves. It is a wave absorbing slot that allows electromagnetic waves on one side to be separated from the other. Another design of the four elements is the structure that consists of four orthogonal radiators. The four feed-through pair antenna elements are located orthogonal to each other for best isolation and provide vertical and horizontal polarization. The two configurations have a broad bandwidth $(640 \mathrm{MHz}$ and $400 \mathrm{MHz}$ ), high efficiency (over $75 \%$ ), good isolation (>-20 dB), low correlation coefficient $(<0.01)$, high channel capacity $(22.5 \mathrm{bps} / \mathrm{Hz})$, and stable gain characteristics. The proposed MIMO antenna is simple, generalized, and can be adapted for a broad range of frequencies. It was found that there is a close agreement between the measured/calculated antenna parameters and experimental performance.
\end{abstract}

Keywords: Linear polarization, Circular polarization, Compact MIMO antennas, 5GHz applications, Stable gain.

\section{Introduction}

Wireless networking now demands greater bandwidth and reliable connection to meet the widespread use of online video streaming and multimedia devices. A new multi-input multi-output (MIMO) technique has recently been developed to overcome this perceived problem [1,2]. The number of antenna components increases the range of the wireless communication system, the amount of data it receives, and the communication precision. Spreading a more significant number of antenna components over the receiver area improves its effectiveness. However, the terminals' available limited space will increase the terminals' need to connect to each other. Because the components share a high degree of communication, there would be a mutual coupling with multiple antennas positioned close together. The high reciprocal inductance will result in high impedance mismatch, low radiation transfer and limited temperature spread. Therefore, an increased level of isolation in compressed MIMO is necessary for modern wireless applications [3].

A multiple-input multiple-output antenna with polarization diversity can correctly overcome the mutual coupling problem and improve spatial isolation in multi-user MIMO systems. By positioning the radiators horizontally and vertically without incorporating any complex decoupling structure between antenna cells, the correlation between antenna components can be minimized.

Recently, several types of diversity antennas have been released with different radiators [4-10]. A cuboidal polystyrene block with radiating antennas wrapped across four-sides was introduced in [4]. A reconfigurable MIMO antenna, shared aperture (nondirectional) consisting of a horizontal/vertical 
arrangement of radiating components was presented in [5]. The work in [6] was about a three dimension (3D) array composed of eight-antennas with four antennas horizontally and the other vertically. Because the structure of the employed elements in [46] are not planar, it isn't easy to introduce this type of antennas. Printed antennas are more straightforward to integrate into portable systems than antennas on a printed circuit board (PCB). A Two and eight-port MIMO antenna designs have been used in $[7,8]$, respectively, for orthogonally dual-polarized (linear polarization) radiators, while [9] proposed a dual-port multi-band miniaturized radiating elements for diversity effects only. The compact MIMO fourelement dual-band double-sided slot antenna is presented in [10], consisting of four orthogonal slot antennas, each with two radiating elements on the upper and lower substrate layer.

The MIMO antennas' polarization diversity in [410] was shown to have been achieved using linearly polarized antenna components, the ground plane's unit cells is not connecting, leading to unpredictable voltage levels[11]. However, circularly polarized (CP) elements have several significant benefits over linear polarized aspects that lead to improving wave propagation. Antennas, such as $\mathrm{CP}$ antennas, are efficient at minimizing multipath fading and are also resistant to polarization misalignment. In comparison, the frequency of electromagnetic (EM) signals in wireless communication remains constant regardless of the CP antennas' direction [12, 13]. Its features make them suitable candidates for possible wireless communication applications.

Fewer CP antenna designs have been proposed for wireless local area network (WLAN), C-band, and satellite navigation applications in the last decade [14-18]. A two-port circular polarized compact MIMO antenna with decoupling radiators isolated without isolation-enhancing techniques was introduced in [14] for WLAN applications. A compact three-radiator MIMO antenna with both pattern and polarization diversity is proposed in [15], consisting of a chamfered-edge square patch antenna and two printed dipoles. It is suggested and examined in [16] a quad-port circularly polarized MIMO antennas system that implement a two-layer frequency selective surface (FSS) superstrate based on planar crossed dipole metal strips. That will make it difficult to combine this with $\mathrm{MM}$ integrated circuits in mobile devices. A 4-port linear and circular polarized MIMO antenna trimmed in corners with tri-band frequency operation covering global system for mobile communications (GSM), WiMAX and WLAN bands has been developed and studied in [17]. The MIMO antennas proposed in [14-17] produce either left hand or right hand circularly polarized waves. Further, in [18], two configurations of 4-port dual-circular polarized MIMO antenna radiate left-hand circular polarization (LHCP)/ righthand circular polarization (RHCP) waves. The MIMO antennas' polarization diversity in [14-18] was shown to have been achieved using a circularly polarized antenna.

In this paper, the MIMO antenna system's design, based on two configurations with compact radiation elements and high isolation, is presented for $5 \mathrm{GHz}$ and C-band applications that include both linear and circular polarization. The proposed design could be adapted to any resonance frequency by adjusting a few parameters. One of the configurations contains four radiation elements of rectangular-slot resonator deployed at various corners of the circuit board to exhibit mutually orthogonal polarizations, with the pattern and polarization diversity. The MIMO antenna produces a complete RF radiation pattern and diversity between each side of the PCB for $5 \mathrm{GHz}$ applications. The other antenna design is based on a dual-port dual-rectangular tapered on a truncated circular antenna, which gives a symmetrical radiation pattern and conveniently fabricates to provide LHCP/RHCP and can be used to create a 2 x 2 MIMO antenna for C-band. It is important to note that the MIMO antenna cannot be used for real-time operation when the ground plane for the unit cells is not common, resulting in unpredictable voltage levels [11]. In this work, to provide a reference plane for practical applications, the unit cells' ground planes are connected. The two configurations have a compact size with low inter-element separation and high envelope correlation coefficient. The simulated and measured findings are used to show the feasibility of the suggested concept technique.

The following matters are discussed in this paper. Section 2 defines the topology of the proposed singleelement circular-polarized antenna, while section 3 shows the single-element linear-polarized antenna. Sections 4 and 5 present the complete configuration and performance of the MIMO antenna system. A comparison with the referenced antennas is provided in section 6. The conclusion is finally stated in section 7.

\section{Single-element circular-polarized antenna}

Fig. 1 demonstrates the topology of the proposed dual-port CP antenna, designed using the Computer Simulation Technology (CST) studio suite. This viafed circularly polarized antenna has been created on a low cost FR4 dielectric substrate of thickness 1.6 $\mathrm{mm}$, the relative permittivity of 4.3 , and 0.025 loss 
tangent. The compact size of the antenna is about $12.7 \times 12.7 \times 1.6 \mathrm{~mm}^{3}$.

In the truncated circular patch, a $0.36 \mathrm{~mm}$-wide slot is cut. The U-slot aims to introduce circular polarization and capacitance so that the inductance caused by the feeding probe can be eliminated to improve bandwidth and impedance. The feed probe is attached to the launcher under the ground plane.

The approximate value of the equations associated with the model's transmission line is calculated by evaluating them as [19] and approximating the results. The design calculation is based on how the circle patch antenna will be made. The antenna parameter values and the parameters of the MIMO array antenna are reported in Table 1. Based on the design Equation (1) of circular patch antennas, the radius determined to be approximately $12.7 \mathrm{~mm}$

$$
a=\frac{F}{\sqrt{\left\{1+\frac{2 h}{\pi \varepsilon_{r} F}\left[\ln \left(\frac{\pi F}{2 h}\right)+1.7726\right]\right\}}}
$$

Where

$$
F=\frac{8.791 \times 10^{9}}{f_{r}\left(\sqrt{\varepsilon_{r}}\right)}
$$

$\varepsilon_{r}$ : Dielectric constant of substrate, h: Height of substrate, a: Radius, and $f_{r}$ : Resonant frequency.

The fringing effect is not taken into consideration in Equation (1). The effective radius $\left(a_{e}\right)$ of the patch is used as fringing makes the patch electrically larger as given by [19]

$$
a_{e}=\frac{a}{\sqrt{\left\{1+\frac{2 h}{\pi \varepsilon_{r} a}\left[\ln \left(\frac{\pi a}{2 h}\right)+1.7726\right]\right\}}}
$$

The frequency of the proposed circular patch is defined in the following equation

$$
\begin{gathered}
\left(f_{r}\right)_{\mathrm{mn} 0}=\frac{1}{2 \pi \sqrt{\mu \varepsilon}}\left(\frac{\chi^{\prime m n}}{a_{e}}\right) \\
X(t+1)=X_{p}(t)-A . D
\end{gathered}
$$

Where; $\chi^{\prime m n}$ represents the zeroes of the derivative of the Bessel function, $\mu$ : permeability coefficient, and $\varepsilon$ : material permittivity In Fig. 1, the antenna's feed-line location is located towards the right and left side of the upper U-slot to obtain LHCP and RHCP, respectively. Fig. 2 displays the simulated reflection coefficients tuned at $6.16 \mathrm{GHz}$ and achieved less than $-19 \mathrm{~dB}$. The

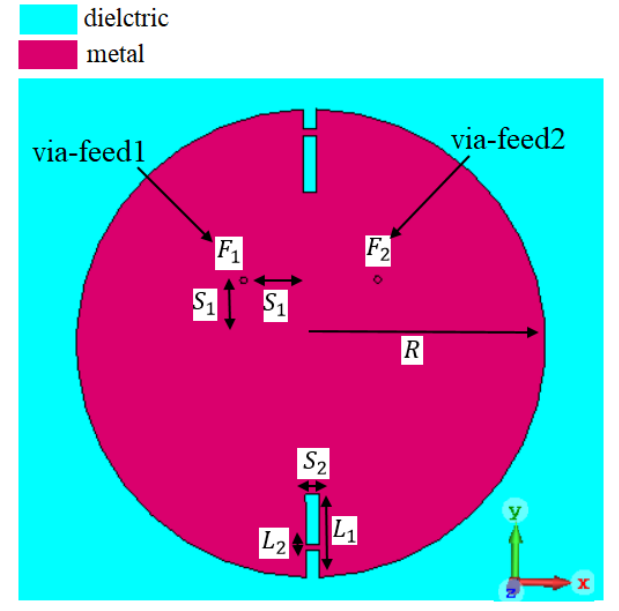

Figure. 1 Front view of dual-CP

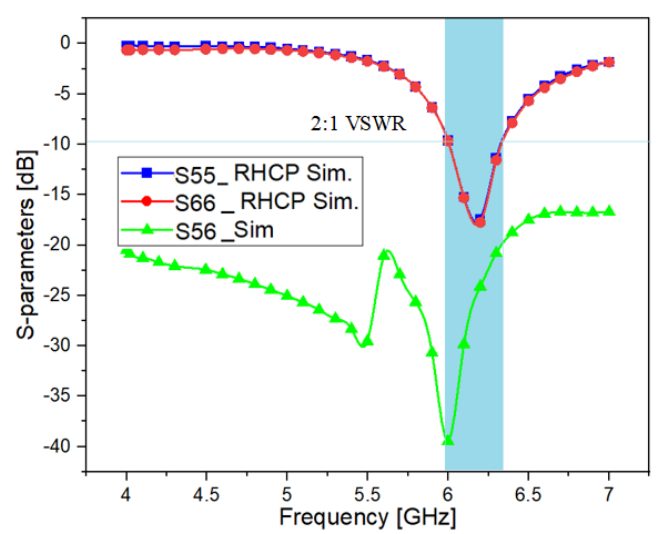

Figure. 2 Reflection coefficients of dual-CP

Table. 1 Parameters dimension of the designs in $\mathrm{mm}$

\begin{tabular}{|c|l|c|l|c|l|}
\hline Param. & value & Param. & value & Param. & value \\
\hline$L$ & 19.9 & $W$ & 15.3 & $S h_{1}$ & 4.66 \\
\hline$L_{f 1}$ & 7.5 & $W_{f 1}$ & 4.32 & $S h_{2}$ & 19 \\
\hline$L_{f 2}$ & 5.5 & $W_{f 2}$ & 1 & $g l_{1}$ & 5.2 \\
\hline$L_{f 3}$ & 6.9 & $W_{f 3}$ & 13.58 & $g l_{2}$ & 16.5 \\
\hline$L_{1}$ & 2.7 & $\mathrm{R}$ & 12.7 & $g l_{3}$ & 16.28 \\
\hline$L_{2}$ & 0.2 & $S_{1}$ & 2.18 & $g w_{1}$ & 12.3 \\
\hline$L_{\text {sub }}$ & 60 & $W_{\text {sub }}$ & 60 & $g w_{2}$ & 9.7 \\
\hline & & & & $S_{2}$ & 0.36 \\
\hline
\end{tabular}

bandwidth of 10-dB impedance is about $0.322 \mathrm{GHz}$. Furthermore, the transmission coefficient is less than $-22 \mathrm{~dB}$. It is evident from the $3 \mathrm{D}$-antenna pattern in Fig. 3 that the designed antenna emit right-hand $C P$ waves in the positive $\mathrm{z}$ direction (z-y plane) while its mirror image radiates left-hand CP waves in the positive $z$ direction ( $z$-x plane). The 2D-pattern on the proposed design was clearly verified that it presents RHCP and LHCP polarities as shown in the Fig. A horizontal main beam in Fig. 3 rotates anti-clockwise to confirm the RHCP activity of the unit cell. Moving on the opposite side, the main beams in the unit cell 


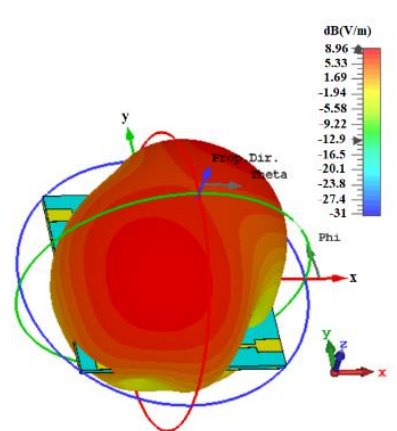

(a)

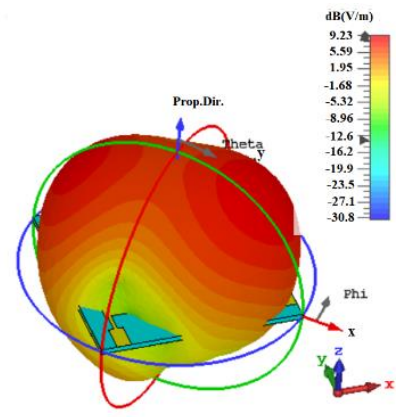

(b)

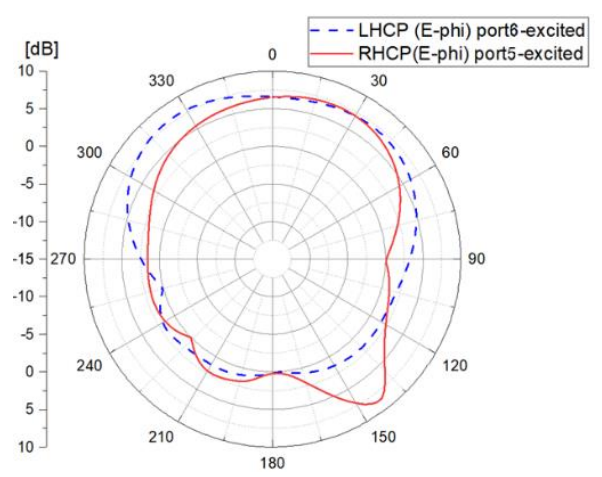

(c)

Figure. 3 Radiation pattern of the dual-CP: (a) 3-D RHCP (b) 3-D LHCP and (c) 2-D pattern

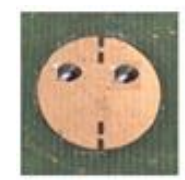

(a)

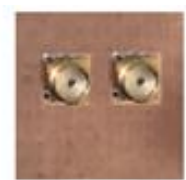

(b)
Figure. 4 Fabricated dual-CP: (a) front and (b) back view

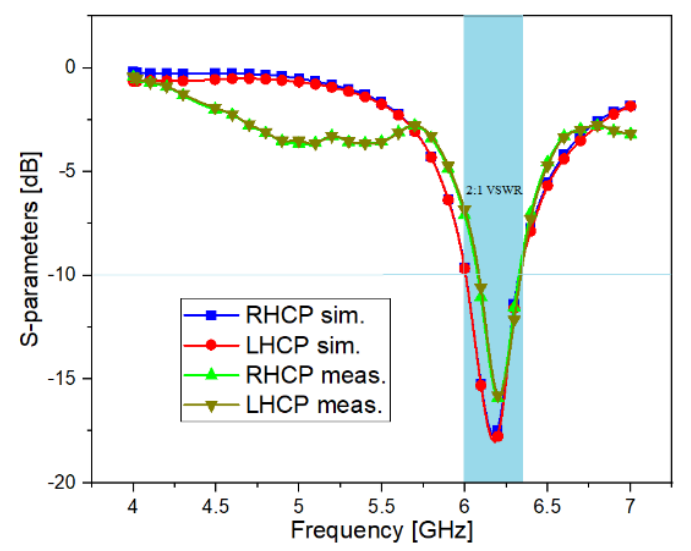

Figure. 5 Measured S-parameters of RHCP and LHCP

rotate clockwise to validate the operation of the LHCP.

An antenna element has been fabricated and tested successfully. Fig. 4 shows the front/back view of the prototype sample. The model's S-parameters are tested, compared and illustrated as shown in Fig.
5. The fabricated sample was found to be functioning very close to the simulation.

\section{Single-element linear-polarized antenna}

The main properties of a single element rectangular-slot resonator with different design parameters have been examined in this section, and the simulation and measurement results are discussed. Fig. 6 displays the front and back view of the antenna configuration. As shown, the antenna's design consists of a rectangular-slot in the ground plane with a rectangular microstrip resonator used at the top of the slot resonator.

The antenna has a compact profile of $\mathrm{W} \times \mathrm{L}$, and is made of an FR4 dielectric with a relative substrate' permittivity of 4.3, 0.025 tangent loss and a thickness of $1.6 \mathrm{~mm}$. The antenna parameter values are reported in Table 1. The width of the patch is measured using the patch width calculation formula:

$$
W=\frac{C}{2 f_{r}} \sqrt{\frac{2}{\varepsilon_{r}+1}}
$$

Where, $\varepsilon_{r}$ : Value of the dielectric layer, $W$ : Width of the patch, $C$ : Speed of light, and $f_{r}$ : Resonance frequency.

The configurations of the rectangular-slot with the final design are shown and compared in Fig. 7. As shown, the antenna's output depends on the feed-line location relative to the radiator, and the resonance occurs when the feed-line is in the middle. The prototype introduces good impedance matching and sufficient isolation at the target frequency with broad bandwidth.

The results obtained indicate that the singleelement antenna is equipped with an impedance bandwidth of 1.14 and $0.6 \mathrm{GHz}$ for $\mathrm{S} 11 \leq-6 \mathrm{~dB}$ and $\mathrm{S} 11 \leq-10 \mathrm{~dB}$, respectively. Besides, at the antenna resonance frequency, the feature of mutual coupling of the design is lower than $-20 \mathrm{~dB}$.

\section{MIMO antenna design}

The front and back schematics of the MIMO antenna configuration for WLAN/WiMAX and CBand are shown in Fig. 8 (a-b). The design has been fabricated on FR4 substrate (with details of permitivity $=4.3$ and loss tangent $=0.025$ ) with the size of $60 \times 60 \times 1.6$. A linear-polarized $4 \times 4$ MIMO antenna is positioned orthogonal to each other to transmit and receive the signals in both horizontal and vertical directions. Four pairs of dual-polarized radiators have been mounted at various corners of the 


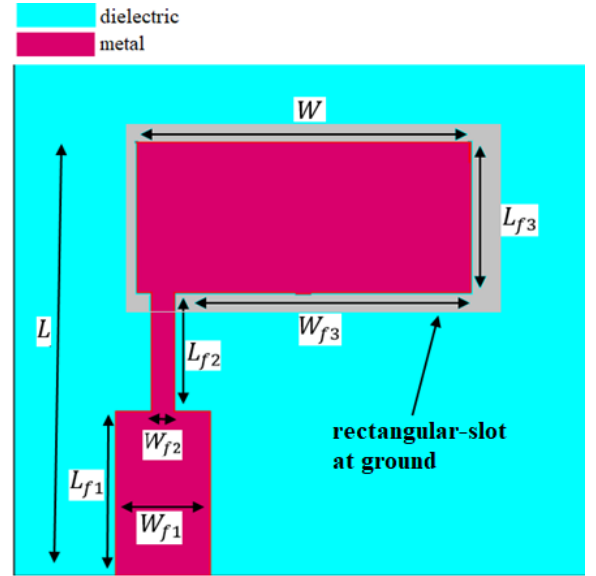

Figure. 6 Front and back view of LP unit cell.

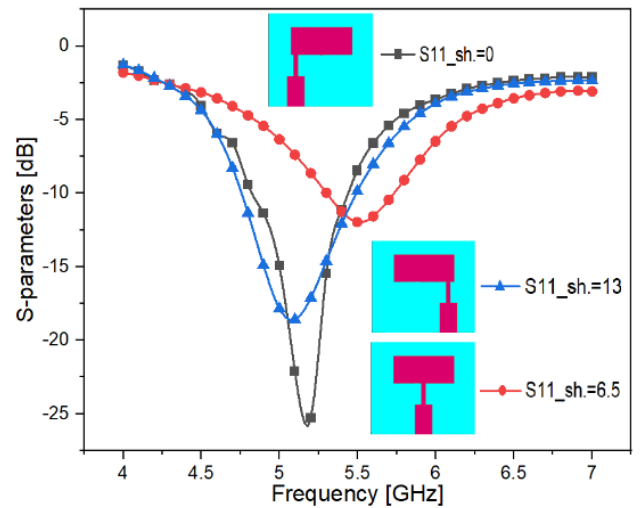

Figure. 7 Configurations of feed line

$\mathrm{PCB}$, as shown. Every couple of microstrip antenna will induce orthogonal polarizations to boost the design's MIMO performance [20]. Furthermore, a dual-port CP MIMO antenna configurations designed for C-Band applications to radiate RHCP and LHCP waves when ports-5 and port-6 are excited, respectively.

As observed in Fig. 9(a-c), the MIMO system design has improved isolation characteristics. Therefore, the antenna's isolation characteristics are slightly below $-20 \mathrm{~dB}$ and exhibit good results covering 4.8-5.44 GHz during the operating bandwidth of the antenna. These features can be done with no loss of purpose. The isolation of the antenna should be as minimal as possible to optimize the provision for small devices. The design is easy to minimize production costs and cut down the manufacturing time needed to produce an antenna.

The overall comparative performances of a 1-4 elements antenna are shown in Fig. 9. Robust impedance matching is observed for all of the components, as mentioned earlier.

The top and bottom views of the proposed prototype are shown in Fig. 10(a-b), respectively. The MIMO antenna system is mounted on a low-cost 1.6

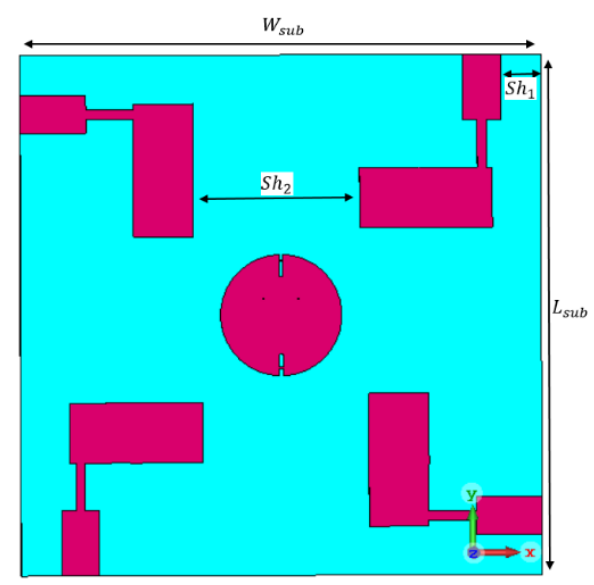

(a)

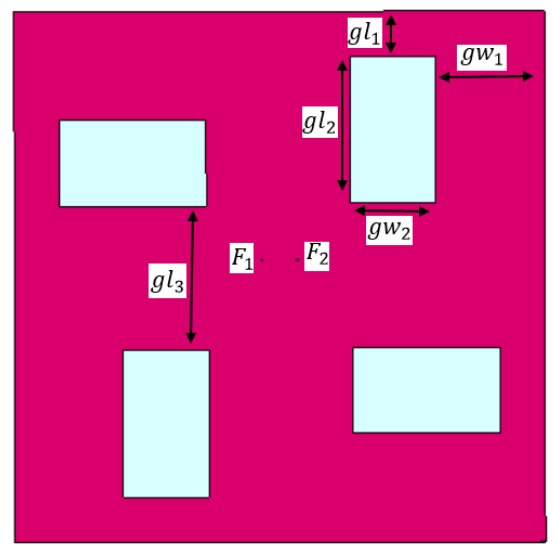

(b)

Figure. 8 Linear and circular MIMO antenna configuration: (a) front and (b) back view

mm FR4 substrate with a size of $60 \times 60 \mathrm{~mm}^{2}$. Its characteristics in terms of S-parameters, radiation patterns and levels of gain have been correctly measured.

For calculating the antenna outcomes, it should be noted that one port remained excited while others were loaded with 50 loads. The simulated/measured S-parameters (S11-S44 and S12-S15) of the diversity antenna radiators are shown in Fig. 9(a-b). It can be observed that strong S-parameters with a very high impedance bandwidth $(\mathrm{S} 11<10 \mathrm{~dB}$ within $4.7-5.9 \mathrm{GHz})$ and low mutual coupling $(\mathrm{S} 21<-20 \mathrm{~dB})$ are provided by the rectangular-slot diversity antennas. There is some deflection from the measurements and simulations that may be primarily due to mistakes in the manufacture, feeding, and calculation processes.

\section{MIMO antenna system performance}

Evaluation of the potential MIMO performance of the antenna system is based on envelop correlation coefficients (ECC), mean effective gain (MEG), channel capacity, and channel capacity loss (CCL). 


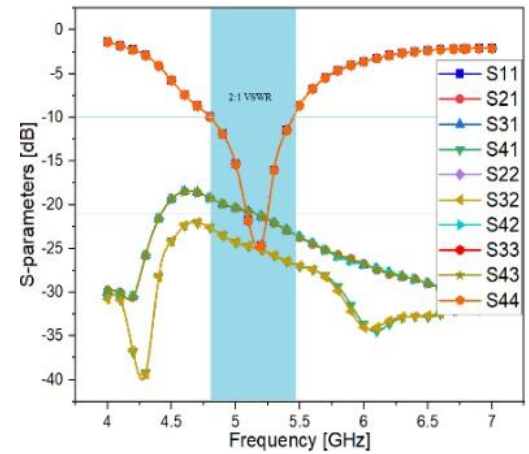

(a)

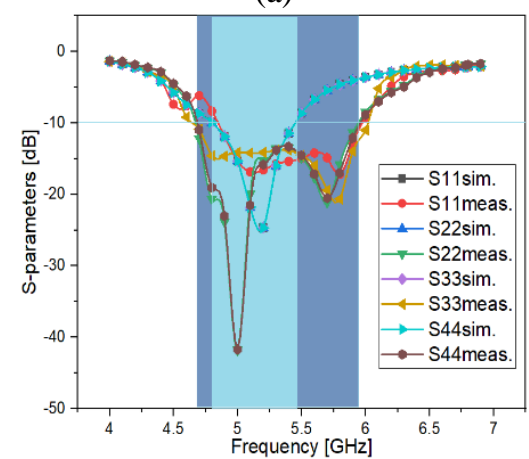

(b)

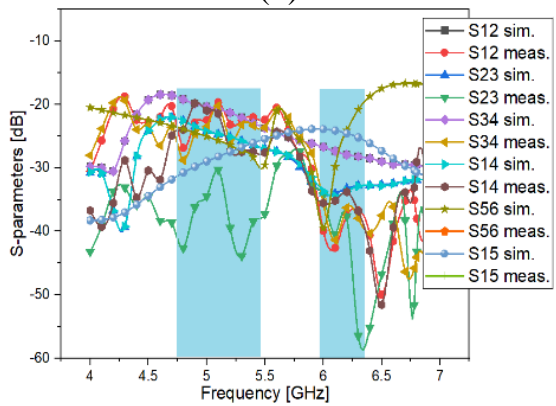

(c)

Figure. 9 Simulated and measured reflection Coefficients: (a) sim. of Ref. and Trans. Coeff. (b) Sim. and meas. of Ref. Coeff. (c) Sim. and meas. of Trans. Coeff

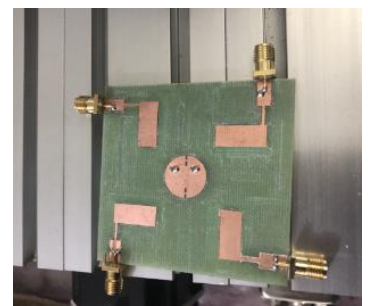

(a)

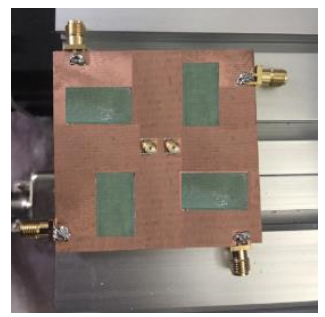

(b)
Figure. 10 Proposed prototype: (a) Top view (b) Bottom view

The ECC is usually used to evaluate the multiantenna system's diversity, where the low ECC value means higher isolation and greater diversity gain. The ECC value should usually be below 0.5 so that it is possible to achieve good diversity characteristics for mobile terminal applications [21]. Since ECC's exact formula for diversity antenna, for brevity, has already

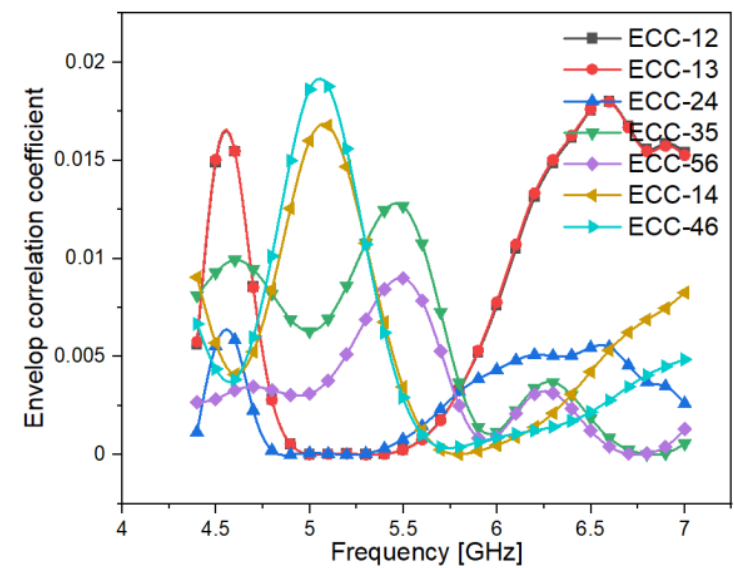

Figure. 11 Envelop correlation coefficient

been discussed in [22], it is not mentioned. The ECC function determined by the scattering parameters is shown in Fig. 11. Its values for the entire operating band are less than 0.02 , which satisfies an appropriate ECC $(<0.5)$ requirement for MIMO operations.

The channel capacity in Fig. 12 is accomplished by providing the same power to be assigned to each antenna at the transmitter, and Rayleigh channel is the propagation scenario, with $20 \mathrm{~dB}$ SNR at the end of the receiver and an average fading of over 1000 Rayleigh in the orthogonally-polarized antenna system [23].

The proposed MIMO system's capacity is 22.5 $\mathrm{bps} / \mathrm{Hz}$ through the operating band, and the channel capacity reached $22.1 \mathrm{bps} / \mathrm{Hz}$ for an ideal $4 \times 4$ MIMO antenna. The expected achievable data throughput can reach a speed of up to 14.4-Gbps (20$\mathrm{dB}$ SNR) within the 4.8-5.44 GHz band when multiplied by the total bandwidth $(640 \mathrm{MHz})$. The orthogonal polarization technique, therefore, showed an excellent channel capacity close to the ideal case $(22.1 \mathrm{bps} / \mathrm{Hz}$ ) and 4.7 times the conventional SISO system as shown in Fig. 12.

In theory, by increasing the number of MIMO elements, the channel capacity could be improved. However, uncorrelated Rayleigh deterioration leads to the MIMO system's channel capacity loss (CCL). The loss of capacity $\left(C_{\text {loss }}\right)$ for the MIMO antennas can be computed by using the following equation [24]:

$$
C_{\text {loss }}=-\log _{2} \operatorname{det}\left(\psi^{R}\right)
$$

Where $\psi^{R}$ is the matrix of correlation of the receiving antenna given by:

$$
\psi^{R}=\left[\begin{array}{ccc}
\rho_{11} & \cdots & \rho_{18} \\
\vdots & \ddots & \vdots \\
\rho_{81} & \cdots & \rho_{88}
\end{array}\right]
$$




$$
\begin{gathered}
\rho_{i i}=1-\left|\sum_{m=1}^{8} S_{i m}^{*} S_{m i}\right|, \text { and } \\
\rho_{i j}=-\left|\sum_{m=1}^{8} S_{i m}^{*} S_{m j}\right|, \text { for } i, j=1, \ldots, 8
\end{gathered}
$$

Fig. 13 shows a simulated loss in the designed antenna system's capacity, where the simulated losses of capacity in an operating frequency band are less than $0.4 \mathrm{bps} / \mathrm{Hz}$, an acceptable CCL limit [25].

The MEG ratios $\left(\left|M E G_{i} / M E G_{j}\right|<3 d B\right)$ are calculated to evaluate the various propagation pathways' imbalanced rates to quantify the mean received signal intensity for each radiator [26]. The antenna MEG can be calculated by the 2D gain pattern provided by [27]. As can be seen from Table 2, the MEG for antennas (Ant.1-Ant.4) is varied from -6.80033 to $-6.20536-\mathrm{dBi}$. Consequently, the MEG variations over the entire operating bandwidth are around 1-dB lower, which is much smaller than the accepted standard $\left(\left|M E G_{i} / M E G_{j}\right|<3 d B\right)$.

A good balance of power and a low loss of diversity can, therefore, be ensured. Therefore, the proposed MIMO diversity array can provide desirable performance in the MIMO system based on the calculated ECC, MEG, CCL, and channel capacity as described above.

In Fig. 14(a-d), the simulated and measured radiation patterns of the four-element antennas array

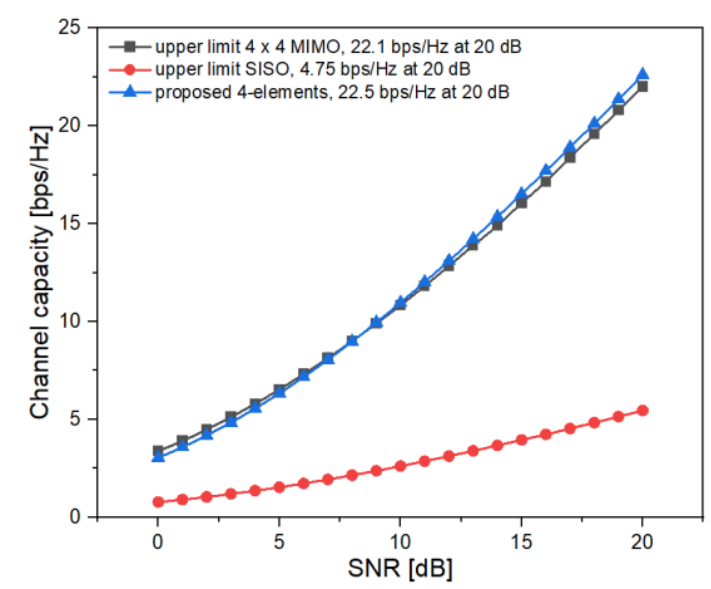

Figure. 12 Ergodic channel capacities

Table 2. Calculated MEG from two-dimensional gain

\begin{tabular}{|c|c|c|c|c|}
\hline $\begin{array}{c}\text { Freq. } \\
\text { [GHz] }\end{array}$ & MEG1 & MEG2 & MEG3 & MEG4 \\
\hline 5.0 & -6.47001 & -6.4702 & -6.47024 & -6.47004 \\
\hline 5.15 & -6.20538 & -6.20548 & -6.20552 & -6.20536 \\
\hline 5.4 & -6.7976 & -6.80033 & -6.80024 & -6.79761 \\
\hline
\end{tabular}

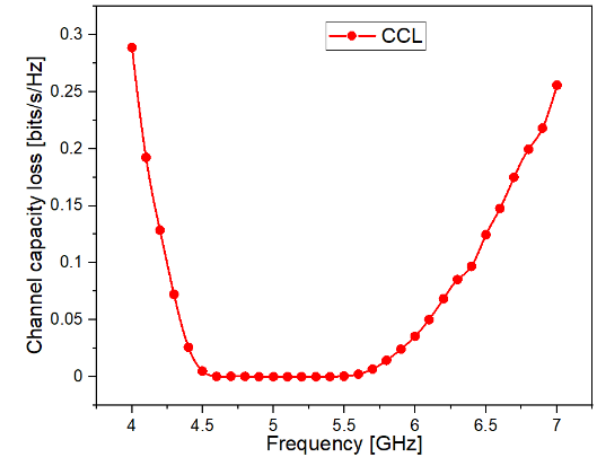

Figure. 13 Channel capacity loss of the proposed MIMO antenna

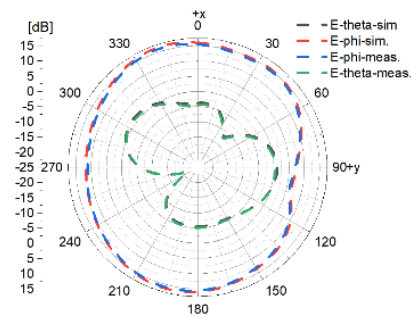

(a)

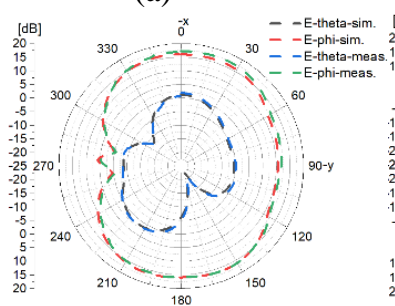

(c)

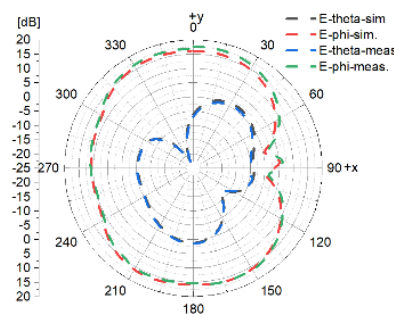

(b)

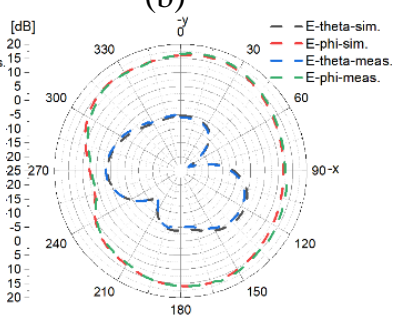

(d)
Figure. 14 The proposed MIMO system's radiation patterns in the xy plane: (a) Ant.1 (b) Ant.2 (c) Ant.3 (d) Ant. 4

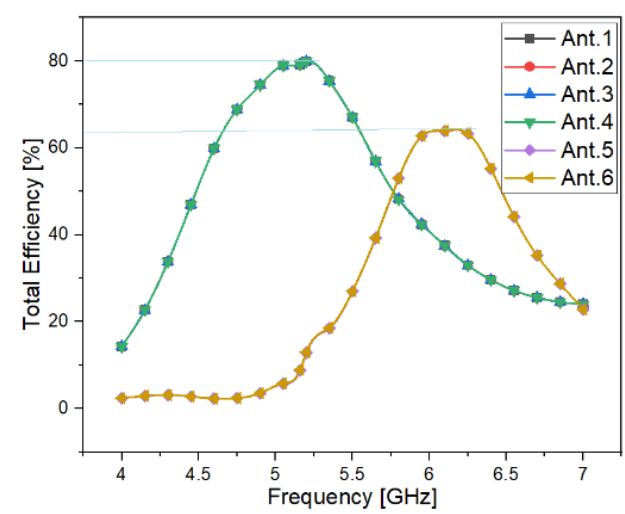

Figure. 15 Total antenna efficiency for LP and CP

for the xy-plane show that the orthogonally element shave quasi-omnidirectional radiation patterns that primarily cover all sides of the PCB board.

As can be seen, each side of the mainboard is filled with radiation patterns that are variously vertical and horizontal in polarization. Because of 
Table 2. Comparison the work in progress with the antennas referenced

\begin{tabular}{|c|c|c|c|c|c|c|c|c|}
\hline Ref. & $\begin{array}{c}\text { BW. } \\
(\mathrm{GHz})\end{array}$ & $\begin{array}{l}\text { Eff. } \\
(\%)\end{array}$ & $\begin{array}{c}\text { Isolation } \\
(\mathrm{dB})\end{array}$ & board Size & $\mathrm{ECC}$ & Applications & $\begin{array}{c}\text { No. of } \\
\text { elements }\end{array}$ & $\begin{array}{c}\mathrm{LP}, \mathrm{CP} \\
\text { polar. }\end{array}$ \\
\hline [9] & $\begin{array}{l}2.2-2.6 \\
3.2-3.7\end{array}$ & --- & $>12.5$ & $45 \times 90$ & $<0.001$ & $\begin{array}{c}\text { WLAN } \\
\text { WiMAX }\end{array}$ & $2 \times 2$ & LP \\
\hline [7] & $3-12$ & --- & $>15$ & $35 \times 35$ & $<0.3$ & UWB & $4 \times 4$ & LP \\
\hline [3] & $5.6-5.8$ & 70 & $>15$ & $26 \times 26$ & $<0.2$ & WLAN & $4 \times 4$ & LP \\
\hline [10] & $\begin{array}{c}2.46 \\
3.5\end{array}$ & --- & $\begin{array}{l}24 \\
24\end{array}$ & $60 \times 60$ & $\begin{array}{l}<0.02 \\
<0.07\end{array}$ & $\begin{array}{c}\text { WLAN } \\
\text { WiMAX }\end{array}$ & $4 \times 4$ & LP \\
\hline [15] & $5.5-6.25$ & $\begin{array}{l}50- \\
85\end{array}$ & $>15$ & $29 \times 48$ & $<0.01$ & WLAN & $3 \times 3$ & LP \\
\hline [17] & $\begin{array}{c}1.7-1.88, \\
3.3-3.7 \\
5.12-5.37\end{array}$ & --- & $>37$ & $165 \times 165$ & --- & $\begin{array}{c}\text { GSM,WiMAX, } \\
\text { WLAN }\end{array}$ & $4 \times 4$ & $\mathrm{LP}, \mathrm{CP}$ \\
\hline [11] & $3.7-8.8$ & --- & $>12$ & $32 \times 32$ & $<0.4$ & $\begin{array}{c}\text { WLAN } \\
\text { WiMAX }\end{array}$ & $2 \times 2$ & $\mathrm{CP}$ \\
\hline$[18]$ & $3.4-3.8$ & --- & $>19$ & $60 \times 60$ & $<0.12$ & Sub-6 GHz & $4 \times 4$ & $\mathrm{CP}$ \\
\hline [8] & $3.3-3.9$ & $\begin{array}{c}60- \\
80 \\
\end{array}$ & 18 & $150 \times 75$ & $<0.005$ & Sub-6 GHz & $8 \times 8$ & LP \\
\hline $\begin{array}{l}\text { Present } \\
\text { work }\end{array}$ & $\begin{array}{c}4.8-5.44 \\
5.9-6.3\end{array}$ & $\begin{array}{c}62- \\
80\end{array}$ & $\begin{array}{l}>20 \\
>20\end{array}$ & $60 \times 60$ & $<0.01$ & $\begin{array}{l}\text { WLAN, } \\
\text { WiMAX } \\
\text { C-Band }\end{array}$ & $\begin{array}{l}4 \times 4 \\
2 \times 2\end{array}$ & $\mathrm{LP}, \mathrm{CP}$ \\
\hline
\end{tabular}

that, the antenna exhibited very good coverage and polarization diversity, and its potential is explored for future wireless applications.

Furthermore, high efficiencies with slight variations are achieved within the range of 4.8-5.44 $\mathrm{GHz}$, as shown in Fig. 15, more than $78 \%$ efficiencies have been performed for the elements (Ant.1-4) and more than 60\% for the elements (Ants.5-6) of the MIMO design at 5.15 and $6.13 \mathrm{GHz}$, respectively.

\section{Comparison with antennas referenced}

Table 3 compares the proposed work with the references considered. It can be seen that the proposed tow configurations provide better results compared to the references considered. The current design has broad bandwidth $(640 \mathrm{MHz}, 400 \mathrm{MHz})$ compared to previous methods, high efficiency (over $75 \%)$, good isolation $(>-20 \mathrm{~dB})$, low profile size $(60$ x $60 \mathrm{~mm}^{2}$ ), low correlation coefficient $(<0.01)$, high channel capacity $(22.5 \mathrm{bps} / \mathrm{Hz})$ as shown in Fig. 12 and linear/circular polarization which is comparable to those of other references shown in Table 3.

\section{Conclusion}

Based on two configurations with a low profile and simple structure of a six-port planner MIMO antenna are designed to provide pattern and polarization diversity. One configuration is a dualport showing circular polarized radiating left-hand $\mathrm{CP}$ and right-hand $\mathrm{CP}$ waves when port 5 and port 6 are excited, respectively. On the other hand, the other MIMO configuration is a quad-elements placed orthogonally to each other to provide linear (Vertical/Horizontal) polarization for $5 \mathrm{GHz}$ applications. The MIMO antennas system provide a compact size $\left(60 \times 60 \mathrm{~mm}^{2}\right)$ dual operating mode where the first mode cover WLAN/Wi-MAX bands (3.4 - 5.44) $\mathrm{GHz}$, whereas the second mode covers the C-band (5.9 - 6.3) GHz. The measured isolations and correlation coefficient of the two modes are better than $-20 \mathrm{~dB}$ and 0.01 , respectively. The agreement between the model simulation and the measured data is evident. The proposed MIMO antenna design method is simple, generalized, and applied to a broad range of frequencies through parametric optimization. Finally, from the results; the proposed two configurations MIMO design is an eligible candidate for WLAN/Wi-MAX and C-band applications since it is compact and versatile and has low coupling over a frequency band of interest.

\section{Conflicts of Interest}

The authors declare that there is no conflict of interest.

\section{Author Contributions}

Q. Hadi devised the idea, the main conceptual ideas and proof outline. M. Jasim was involved in planning and supervised the work. Q. Hadi processed the experimental data, performed the analysis with CST and ADS software, drafted the manuscript and manufactured the prototype and characterized them with 2-port vector network analyzer. M. Jasim aided 
in interpreting the results and worked on the manuscript.

\section{Acknowledgments}

The authors would like to thank Mustansiriyah University (www.uomustansiriyha.edu.iq) Baghdad, Iraq for its support in the present work.

\section{References}

[1] M. A. Jensen and J. W. Wallace, "A review of antennas and propagation for MIMO wireless communications", IEEE Transactions on Antennas and Propagation, Vol. 52, No. 11, pp. 2810-2824, 2004.

[2] D. W. Bliss, K. W. Forsythe, and A. M. Chan, "MIMO wireless communication", Lincoln Laboratory Journal, Vol. 15, No. 1, PP. 97-126, 2005.

[3] S. Pandit, A. Mohan, and P. Ray, "A compact four element MIMO antenna for WLAN applications", Microwave Optical Technology Letter, Vol. 60, No. 2, PP. 289-295, 2018.

[4] M. Bilal, R. Saleem, H. H. Abbasi, M. F. Shafique, and A. K. Brown, "An FSS-Based Nonplanar Quad-Element UWB-MIMO Antenna System", IEEE Antennas and Wireless Propagation Letters, Vol. 16, pp. 987-990, 2017.

[5] M. G. N. Alsath, H Arun, Y. P. Selvam, M. Kanagasabai, S. Kingsly, and R. Natarajan, "An integrated tri-band/UWB polarization diversity antenna for vehicular networks", IEEE Transactions on Vehicular Technology, Vol. 67, No. 7, pp. 5613-5620, 2018.

[6] S. K. Palaniswamy, Y. P. Selvam, M. G. N. Alsath, M. Kanagasabai, S. Kingsly, and S. Subbaraj, "3-D eight-port ultra-wideband antenna array for diversity applications", IEEE Antennas and Wireless Propagation Letters, Vol. 16, pp. 569-572, 2017.

[7] J. Zhu, S. Li, B. Feng, L. Deng, and S. Yin, "Compact dual-polarized UWB quasi-selfcomplementary MIMO/diversity antenna with band rejection capability", IEEE Antennas Wireless Propagation Letter, Vol. 15, pp. 905908, 2016.

[8] N. O. Parchin, Y. I. A. Al-Yasir, H. J. Basherlou, R. A. Abd-Alhameed, and J. M. Noras, "Orthogonally dual-polarised MIMO antenna array with pattern diversity for use in 5G smartphones", IET Microwaves, Antennas and Propagation, Vol. 14, PP. 457-467, 2020.

[9] L. Malviya, M. V. Kartikeyan, and R. K. Panigrahi, "Multi-standard, multi-band planar multiple input multiple output antenna with diversity effects for wireless applications", International Journal of $R F$ and Microwave Computer-Aided Engineering, PP. 1-8, 2018.

[10] W. A. E. Ali, M. I. Ashraf, and M. A. Salamin, "A dual-mode double-sided 4 x 4 MIMO slot antenna with distinct isolation for WLAN/WiMAX applications", Microsystem Technologies, 2020.

[11] D. S. Chandu and S. S. Karthikeyan, "A novel broadband dual circularly-polarized microstripfed monopole antenna", IEEE Transactions on Antennas Propagation, Vol. 65, No. 3, PP. 1410-1415, 2017.

[12] J. Wang, Z. Lv, and X. Li, "Analysis of MIMO diversity improvement using circular polarized antenna," International Journal of Antennas and Propagation, Vol. 2014, pp. 1-9, 2014.

[13] F. A. Dicandia, S. Genovesi, and A. Monorchio, "Analysis of the performance enhancement of MIMO systems employing circular polarization", IEEE Transactions on Antennas Propagation, Vol. 65, No. 9, pp. 4824-4835, 2017.

[14] J. Malik, A. Patnaik, and M. V. Kartikeyan, "Novel printed MIMO antenna with pattern and polarization diversity", IEEE Antennas Wireless Propagation Letter, Vol. 14, pp. 739-742, 2015.

[15] Y. Sharma, D. Sarkar, K. Saurav, and K. V. Srivastava, "Three element MIMO antenna system with pattern and polarization diversity for WLAN applications", IEEE Antennas Wireless Propagation Letter, Vol. 16, pp. 1163 1166, 2017.

[16] M. Akbari, H. Abo Ghalyon, M. Farahani, A. Sebak, and T. A. Denidni, "Spatially decoupling of CP antennas based on FSS for $30-\mathrm{GHz}$ MIMO systems", IEEE Access, Vol. 5, pp. 6527-6537, 2017.

[17] R. S. Parbat, A. R. Tambe, M. B. Kadu, and R. P. Labade, "Dual polarized triple band $4 \times 4$ MIMO antenna with novel mutual coupling reduction approach", IEEE Bombay Section Symposium (IBSS) Mumbai, India, pp. 1-6, 2015.

[18] S. Saxena, B. K. Kanaujia, S. Dwari, S. Kumar, H. C. Choi, and K. W. Kim, "Planar Four-Port Dual Circularly-Polarized MIMO Antenna for Sub-6 GHz Band", IEEE Access, Vol. 8, pp. 90779-90791, 2020.

[19] C. A. Balanis, Antenna Theory: Analysis and Design, John Wiley \& Sons, 2016.

[20] M. K. T. Al-Nuaimi, and W. G. Whittow, "Performance investigation of a dual element IFA array at $3 \mathrm{GHz}$ for MIMO terminals", In: proc. of Antennas and Propagation Conf., Loughborough, UK, pp. 1-5, 2011. 
[21] I. Al-Muttairi and M. J. Farhan, "Frequency reconfigurable monopole antenna with harmonic suppression for internet of things applications", Telecommunication, Computing, Electronics and Control, Vol. 18, pp. 10-18, 2020.

[22] Y. Ban, C. Li, C. Sim, G. Wu, and K. Wong, "4G/5G Multiple Antennas for Future MultiMode Smartphone Applications", IEEE Access, Vol. 4, pp. 2981-2988, 2016.

[23] M. S. Sharawi, M. U. Khan, A. B. Numan, and D. N. Aloi, "A CSRR loaded MIMO antenna system for ISM band operation", IEEE Transactions on Antennas and Propagation, Vol. 61, No. 8, pp. 4265-4274, 2013.

[24] G. Das, A. Sharma, and R. K. Gangwar, "Dual port aperture coupled MIMO cylindrical dielectric resonator antenna with high isolation for WiMAX application", International Journal of $R F$ and Microwave Computer-Aided Engineering, Vol. 27, No. 7, pp. 1-10, 2017.

[25] C. Mao and Q. Chu, "Compact Co-radiator UWB-MIMO Antenna with Dual Polarization", IEEE Transactions on Antennas and Propagation, Vol. 62, No. 9, pp. 4474-4480, 2014.

[26] R. Mittra, Developments in Antenna Analysis and Design, Institute of Engineering and Technology, 2019.

[27] Y. Ding, Z. Du, K. Gong, and Z. Feng, "A novel dual-band printed diversity antenna for mobile terminals", IEEE Transaction on Antennas Propagation, Vol. 55, No. 7, pp. 2088-2096, 2007. 